\title{
Design and performance of a Near Ultra High Vacuum Helium Ion Microscope
}

\author{
R. van Gastel, ${ }^{*}$ L. Barriss, ${ }^{* *}$ C. Sanford**, G. Hlawacek, ${ }^{*}$ L. Scipioni, ${ }^{* *}$ A.P. Merkle, ${ }^{* *}$ D. \\ Voci, ${ }^{* *}$ C. Fenner, ${ }^{* * *}$ H.J.W. Zandvliet, ${ }^{*}$, and B. Poelsema* \\ * Physics of Interfaces and Nanomaterials, MESA+ Research Institute, University of Twente, P.O. \\ Box 217, NL-7500 AE, Enschede, The Netherlands \\ ** Carl Zeiss NTS LLC, One Corporation Way, Peabody, MA 01920 \\ *** LVestus Energy Inc., 8 Merrill Industrial Drive, Hampton, NH 03842
}

The advent of He Ion Microscopy (HIM) as a new technique to image materials and microstructures has enabled a new look at materials that is based on the interaction of swift light ions with matter, as opposed to that of more commonly used high (and low) energy electrons [1]. Initial Carl Zeiss Orion ${ }^{\circledR}$ He Ion Microscope instruments have demonstrated high-resolution imaging, combined with great surface sensitivity, the ability to neutralize charge very efficiently, and with enhanced materials contrast when ion induced secondary electrons are used for imaging. The use of Rutherford backscattered ions to form images has provided a new imaging modality that emphasizes differences in elemental composition and it can also be used to probe samples in-depth.

The HIM provides obvious benefits in terms of novel modes of contrast, surface sensitivity, lateral resolution, depth of field and charge compensation. To achieve ultimate performance, the chamber vacuum of the existing platform may be improved. For instance, carbon deposits due to beam interaction are readily seen due to the surface sensitivity of the technique. At sufficiently high current densities the sharply focused He ion beam may very efficiently decompose or cross-link residual hydrocarbons that are present in the instruments vacuum, more so than an electron beam in a SEM setup. Not only can this obscure a clear view of the sample, thereby negating the benefits of the small spot size, it also limits the available acquisition time for spectroscopic measurements. In addition to this, some materials (Au in particular) have yielded unexpectedly high sputtering rates. On the one hand, this has proven extremely useful in the field of nanopatterning for sensors, plasmonics or other device fabrication applications at the sub-10nm level when operating at high doses. On the other hand, it is undesirable when the instrument is used for materials characterization.

In this presentation we will discuss the basic considerations that went into the design of a Near-UHV (NUHV) Orion Plus ${ }^{\circledR}$ He Ion Microscope, shown in Fig. 1. We will detail how the improved vacuum level is anticipated to alter those processes that are directly relevant to the imaging performance of the instrument such as beam interaction in the surface region and the emission of secondary electrons.

First images recorded with the instrument, an example is shown in Fig. 2, reveal high levels of detail that further emphasize the surface-sensitive imaging characteristics and channeling contrast mechanisms of the helium ion interaction. This will have impact in the areas of surface physics, notably catalysis, corrosion, and other research areas that require increased imaging sensitivity, both laterally and in depth.

References

[1] B. Ward, J. Notte, and N. Economou, J. Vac. Sci. Technol. 24, 2871 (2006). 


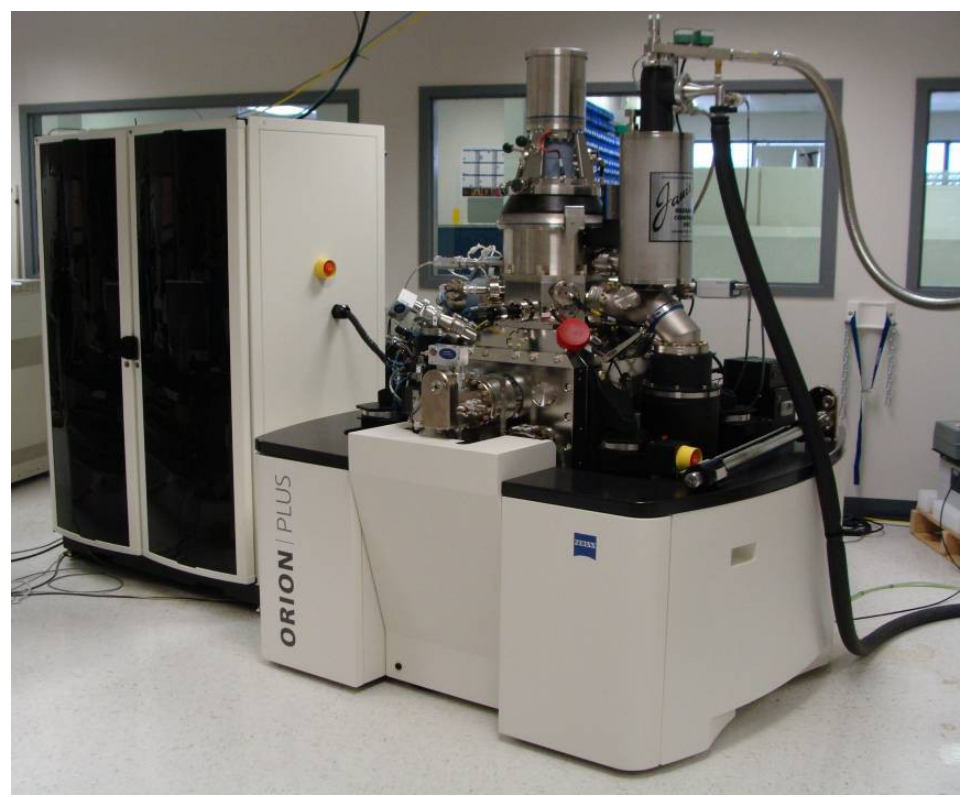

FIG. 1. The completed NUHV Helium Ion Microscope

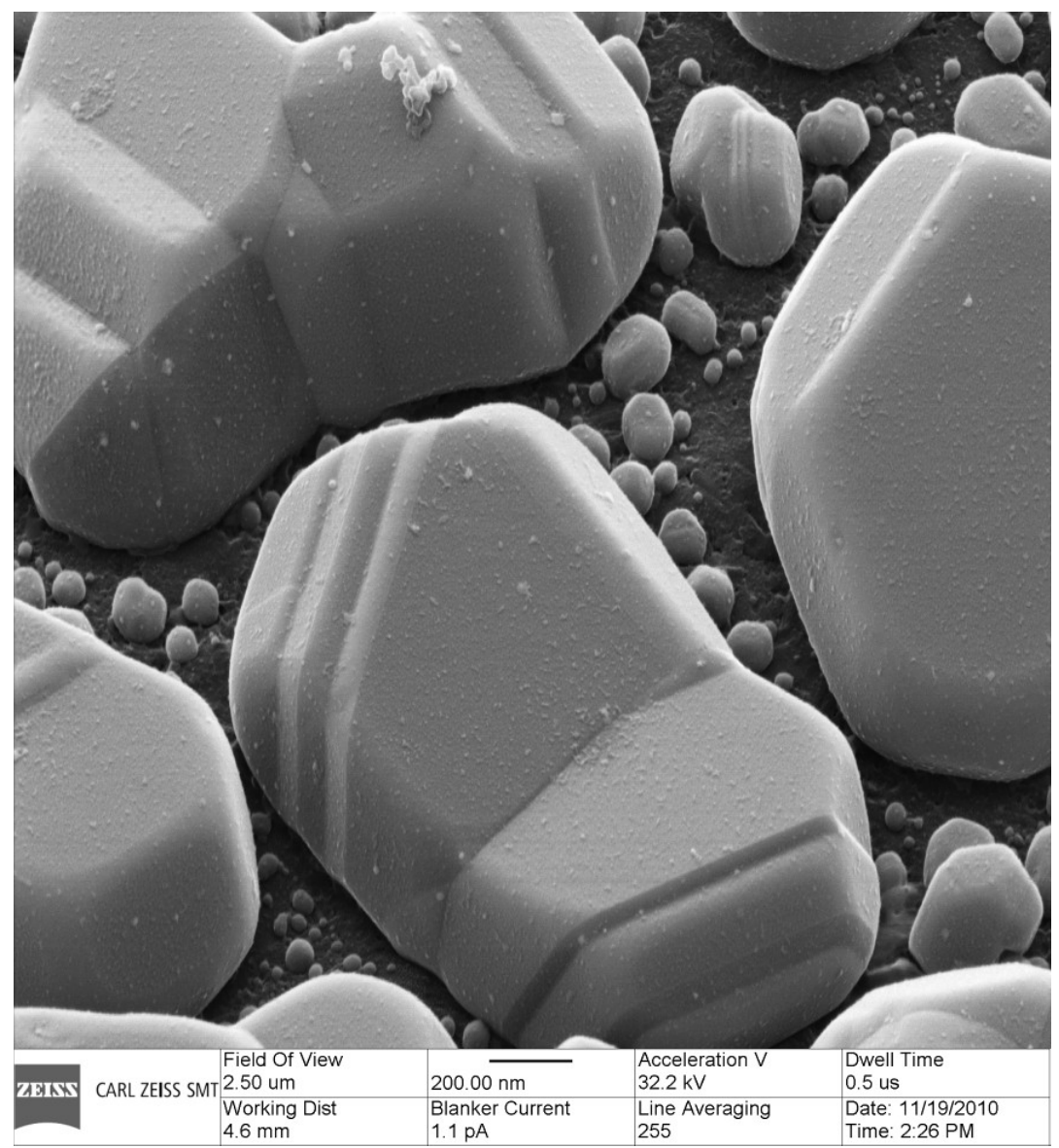

FIG. 2. Initial gold on carbon image from an ultra-high vacuum helium ion microscope. Pronounced surface detail and channeling (grain) contrast is evident in this image, which will be relevant for a number of applications. 